\title{
Author Correction: T cell and antibody responses induced by a single dose of ChAdOx1 nCoV-19 (AZD1222) vaccine in a phase 1/2 clinical trial
}

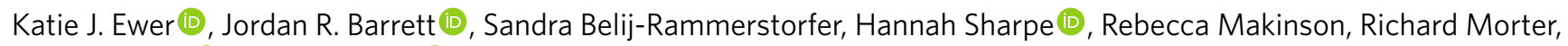
Amy Flaxman (D), Daniel Wright (10, Duncan Bellamy, Mustapha Bittaye, Christina Dold, Nicholas M. Provine, Jeremy Aboagye, Jamie Fowler, Sarah E. Silk, Jennifer Alderson, Parvinder K. Aley, Brian Angus $(D$, Eleanor Berrie,

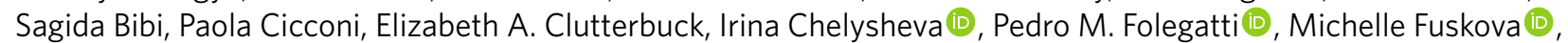
Catherine M. Green, Daniel Jenkin (1), Simon Kerridge, Alison Lawrie, Angela M. Minassian (1), Maria Moore, Yama Mujadidi (D, Emma Plested, Ian Poulton (D, Maheshi N. Ramasamy (1), Hannah Robinson, Rinn Song (1), Matthew D. Snape, Richard Tarrant, Merryn Voysey, Marion E. E. Watson (D), Alexander D. Douglas (1), Adrian V. S. Hill(D, Sarah C. Gilbert (1D, Andrew J. Pollard (1D), Teresa Lambe (i) and the Oxford COVID Vaccine Trial Group*

Correction to: Nature Medicine https://doi.org/10.1038/s41591-020-01194-5, published online 17 December 2020.

In the version of this article initially published, the following names were missing from the author list for the Oxford COVID Vaccine Trial Group: Francesca R. Donnellan, Giacomo Gorini, Gaurav Gupta, Nathifa Moya, Ekta Mukhopadhyay, Fay L. Nugent, Stephannie Salvador, Iman Satti and Iona Jennifer Taylor (all at affiliation 1); Carla Ferreira Da Silva, Natalie G. Marchevsky, Daniel Phillips, Christine S. Rollier, Lisa Stockdale, Adriana Tomic and Marije K. Verheul (all at affiliation 2); Miles W. Carroll (at affiliation 7); and Annina Schmid (at affiliation 9). Also, two author names (Rachel Varughes and Gary Mallett) were incorrect; the correct names are 'Rachel Varughese' and 'Garry Mallett' (respectively). The errors have been corrected in the HTML and PDF versions of the article.

${ }^{\star} \mathrm{A}$ list of authors and their affiliations appears online.

Published online: 21 May 2021

https://doi.org/10.1038/s41591-021-01363-0

๑ The Author(s), under exclusive licence to Springer Nature America, Inc. 2021

\section{Author Correction: Attributes and predictors of long COVID}

Carole H. Sudre, Benjamin Murray (D), Thomas Varsavsky, Mark S. Graham (D), Rose S. Penfold, Ruth C. Bowyer (D, Joan Capdevila Pujol@1 Kerstin Klaser, Michela Antonelli, Liane S. Canas, Erika Molteni@, Marc Modat,

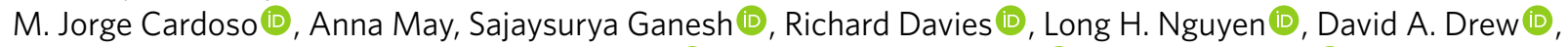

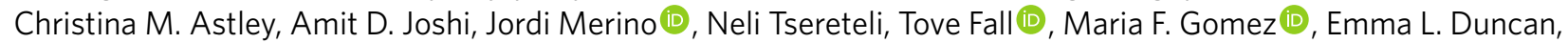

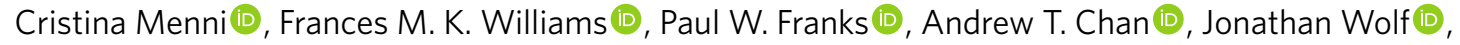
Sebastien Ourselin (1), Tim Spector (1) and Claire J. Steves (1)

Correction to: Nature Medicine https://doi.org/10.1038/s41591-021-01292-y, published online 10 March 2021.

In the version of this article initially published, linkage of the following authors to affiliation 3 (Centre for Medical Image Computing, Department of Computer Science, University College London, London, UK) was incorrect: Benjamin Murray, Thomas Varsavsky, Mark S. Graham, Kerstin Klaser, Michela Antonelli, Liane S. Canas, Erika Molteni, Marc Modat, M. Jorge Cardoso and Sebastien Ourselin. The correct linkage is to affiliation 1 (School of Biomedical Engineering \& Imaging Sciences, King's College London, London, UK). The error has been corrected in the HTML and PDF versions of the article.

Published online: 27 May 2021

https://doi.org/10.1038/s41591-021-01361-2

(c) The Author(s), under exclusive licence to Springer Nature America, Inc. 2021 